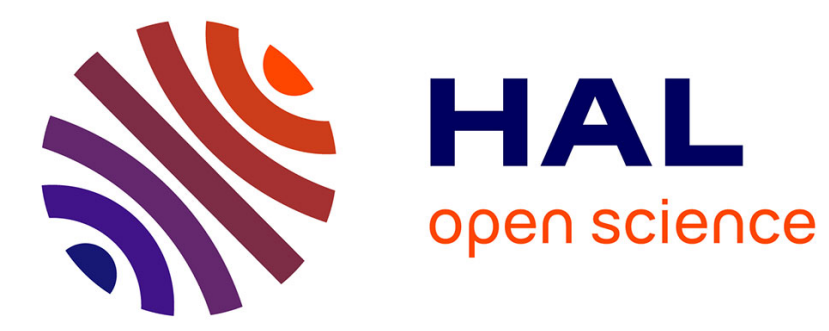

\title{
Investigating swelling-breakdown in double emulsions
}

\author{
Behnam Khadem, Maya Khellaf, Nida Sheibat-Othman
}

\section{To cite this version:}

Behnam Khadem, Maya Khellaf, Nida Sheibat-Othman. Investigating swelling-breakdown in double emulsions. Colloids and Surfaces A: Physicochemical and Engineering Aspects, 2019, pp.124181. 10.1016/j.colsurfa.2019.124181 . hal-02361469

\section{HAL Id: hal-02361469 \\ https://hal.science/hal-02361469}

Submitted on 28 Nov 2020

HAL is a multi-disciplinary open access archive for the deposit and dissemination of scientific research documents, whether they are published or not. The documents may come from teaching and research institutions in France or abroad, or from public or private research centers.
L'archive ouverte pluridisciplinaire HAL, est destinée au dépôt et à la diffusion de documents scientifiques de niveau recherche, publiés ou non, émanant des établissements d'enseignement et de recherche français ou étrangers, des laboratoires publics ou privés. 


\title{
Investigating Swelling-Breakdown in Double Emulsions
}

\author{
Behnam Khadem ${ }^{a}$, Maya Khellaf ${ }^{a}$ and Nida Sheibat-Othman* ${ }^{a}$ \\ ${ }^{1}$ University of Lyon, Université Claude Bernard Lyon 1, CNRS, LAGEP UMR 5007, F-69100, \\ Villeurbanne, France \\ *Corresponding author: nida.otham@univ-lyon1.fr
}

\section{Abstract}

Dilute water-in-oil-in water double emulsions are prepared to investigate the release and swelling phenomena during preparation and storage. During storage, a specific attention is paid to detect the overswelling-breakdown phenomenon that is due to overswelling of the outer droplets which causes their breakage and abrupt release of the inner droplets. A swelling ratio of the outer droplets is defined and is found useful to identify the effect of different parameters on the swelling behavior of the double emulsions. Yet, the investigation of the full droplet size distribution remains necessary. Before overswellingbreakdown, the escape is governed by the Brownian motion of the inner droplets and their coalescence with the external phase through the surface of the outer droplets. The swelling rate is mainly governed by the osmotic gradient between the internal and external water phases.

Keywords: Double emulsions, Overwelling-breakdown, Escape, Release.

\section{Introduction}

To produce double emulsions, typically, a primary emulsion is prepared in a first step under high shear to disperse an internal phase in an intermediate phase. In a second step, this 
primary emulsion is dispersed in an external phase while mixing under a lower shear rate. Both water-in-oil-in-water (W/O/W) and oil-in-water-in-oil (O/W/O) double emulsions can be produced. This two-step method was firstly introduced by Li (1968) [1] for the purpose of extraction $(\mathrm{O} / \mathrm{W} / \mathrm{O})$ and the term liquid membranes was employed. A first hydrophobic phase was emulsified within an aqueous surfactant solution to produce oil droplets coated with a thin membrane of surfactant and water, which was then dispersed into another hydrophobic phase to be washed from solvent by permeation through the membrane. Then, Matsumoto et al. (1976) [2] introduced this method for the formation of double emulsions. A higher shear is employed in the first step in order to produce small inner (i.e., micro) droplets and a lower shear is employed in the second step with the aim of preventing the release of inner droplets and thus bigger outer (i.e., macro) droplets (or globules) are formed. The droplet size of both the inner and outer droplets and the encapsulation efficiency determine the quality of double emulsion products [3].

The preparation and stability of double emulsions have been the subject of many studies. On one hand, during the second preparation step, the outer droplets may undergo breakage, coalescence, and Oswald ripening while the inner droplets may undergo coalescence, growth by Oswald ripening or escape to the external phase $[1,2,4-9]$. On the other hand, during storage, different possible destabilization pathways of the double emulsion may occur, namely inner-inner and outer-outer droplet coalescence, expulsion of the inner droplets (i.e. inner droplet coalescence with the outer phase), Oswald ripening, swelling/shrinkage of the inner (and so of the outer) droplets (i.e. diffusion of water/solvent) and molecular diffusion of dissolved ingredients between the phases $[8,10-$ 19]. The swelling phenomena may ultimately lead to swelling-breakdown, i.e. gradual expulsion of the inner droplets out of the outer droplet [11,19-21], or to a more abrupt 
expulsion here called overswelling-breakdown [11,19-21]. The later phenomenon may cause breakage of the outer droplet together with important escape of the inner droplets. These different phenomena impact the encapsulation efficiency and the droplet size that has a direct influence on the stability of the double emulsions.

Parameters affecting the preparation of double emulsions. During preparation, the operating conditions were found to have the following effects in $\mathrm{W} / \mathrm{O} / \mathrm{W}$ double emulsions: An increase in the encapsulation efficiency was observed when increasing the fraction of the primary emulsion $[2,22,23]$ and the fraction of lipophilic (i.e. internal) emulsifier $[2,24]$. A decrease in the encapsulation efficiency was observed when increasing one of the following parameters: the hydrophilic (i.e. external) emulsifier concentration [2,25], the emulsification time $[5,6,23,24,26-28]$ and the stirring rate (or energy) in the second emulsification step $[5,22,25,27,29,30]$. The effects of increasing the stirring time and rate on the encapsulation efficiency can be explained by an increased outer droplet deformation and breakage $[1,24,27]$ which increases the inner droplet escape rate [28]. The encapsulation efficiency also depends on the sizes of the inner and outer droplets. It increases with bigger outer droplets $[5,9,28,31]$ and with smaller inner droplets or with a reduced inner-to-outer droplet size ratio [32]. A comparative study over various devices (i.e., colloid mill, tooth rim dispersing machine, high pressure homogenizer, and rotating membrane device) indicated the encapsulation efficiency to mainly depend on the outer droplet size but not on the device [9]. The size of the outer droplets is defined by the operating conditions: it was found to decrease for higher stirring rates (or energy) $[5,7,9,31,33,34]$, longer emulsification times $[5,23,28]$ and higher hydrophilic (i.e. external) emulsifier concentrations [25], while bigger outer droplets were produced with higher internal phase fraction $[5,7,34,35]$ and/or higher primary emulsion fraction $[23,33]$. 
Frenkel et al. (1983) [36] introduced a weighted HLB expression to define the effects of both lipophilic and hydrophilic surfactants on the preparation of double emulsions. They outlined that the formation of double emulsion can occur only if both the criteria of (a) weighted-HLB $<10$, and (b) weighted-HLB < desired HLB of the oil phase, are realized, and, the size of the outer droplets should be big enough ( $>5 \mu \mathrm{m}$ in their study) to be able to contain the inner droplets of a certain size. Schmidts et al. (2009 and 2010) [37,38] indicated that, other than the HLB value, the chemical composition of the hydrophilic surfactant needs to be compatible with the oil and lipophilic surfactant type and amount.

Other operating conditions were found to have more complex effects that may appear to be contradictory in the literature. For instance, increasing the internal phase fractions were found to decrease the encapsulation efficiency by $[22,35]$ and to increase it by $[2,5,26]$. This is partly due to the fact that when increasing the inner phase fraction, the concentration of ions also changes. Indeed, ions (e.g. $\mathrm{NaCl}, \mathrm{MgSO} 4$, glucose, dissolved in the inner phase) are usually employed to enhance the stability of the double emulsion, and there concentration and nature were found to affect the characteristics of the system both right after preparation and during storage $[37,38]$. The use of ions was found to have complex effects, where for increasing $\mathrm{NaCl}$ fractions in the inner phase, an increase in the outer droplet size was observed by $[23,39]$ while a decrease was observed by [38]. Similarly, increasing the $\mathrm{NaCl}$ fraction in the inner phase was found to increase the encapsulation efficiency by $[5,23,39]$ but to decrease it by [26]. Increasing the fractions of MgSO4 in the inner phase showed an increase or a decrease depending on the amount of hydrophilic emulsifiers [38].

Parameters affecting the stability of double emulsions during storage. Double emulsions were usually monitored by optical or electronic microscopy during storage in order to 
investigate their evolution during storage in terms of size and number of the inner and outer droplets. The outer droplet size was found to decrease at the beginning of storage which was assumed to be due the release of inner droplets $[16,18]$ and to increase with time which was assumed to be due to droplet coalescence [16]. Ficheux et al. (1998) [40] identified two types of instabilities: inner-inner droplet coalescence and inner droplet coalescence with the outer continuous phase (i.e., escape), and indicated that they were determined by the concentration of hydrophilic surfactant in the external aqueous phase in W/O/W double emulsions. Pays et al. (2001) [12] identified two types of release: molecular release of the encapsulated substance and inner droplet escape by coalescence with the outer phase. They proposed a behavioral curve as a function of the hydrophilic surfactant and inner phase fractions in order to define the border of these two types of release.

Besides inner-inner droplet coalescence, molecular diffusion and inner droplet escape, swelling or shrinkage were also observed to occur in double emulsions containing ions in the inner or outer phases. The first swelling study was reported by Matsumoto and Kohda (1980) [41], regarding water transfer through the oil layer in W/O/W double emulsions. A mechanism of diffusion through the intermediate oil membrane was described by considering the osmotic pressure gradient between the inner and outer water phases as a driving force (i.e., $L_{\mathrm{p}} A_{\mu} \Delta \Pi$ ), where $L_{\mathrm{p}}$ is the permeation coefficient and $A_{\mu}$ the surface of inner droplets. The osmotic pressure gradient is proportional to the ions concentration gradient, $\Delta \Pi \propto \Delta C$.

In subsequent works, the permeation coefficient of the oil phase and the swelling rate were investigated by varying the oil type or viscosity, where a decrease in the swelling rate was observed when increasing the oil viscosity $[10,41-43]$. Increasing the lipophilic surfactant 
concentration (i.e. internal emulsifier) showed an increase in the swelling rate in some works $[11,15,44]$ and a decrease in others [41]. The swelling rate was found to be higher with higher osmotic pressure gradients $[15,41,43]$ and lower for higher inner phase fractions [15].

Terrisse et al. (1994) [45], observed that droplet swelling was followed by a rupture in the oil membrane, which was later called swelling-breakdown [11]. This phenomenon consists of the expulsion of inner droplets, through their coalescence with the outer phase. As it is enhanced by swelling, and may lead to the breakage of the outer droplet. This behavior was also observed by Raynal et al. (1993) [21] who indicated that it can be tuned through the osmotic pressure gradient. Jager-Lezer (1997) [11] and Geiger et al. (1998) [20] reported that the swelling-breakdown was the main cause of release in their case while the diffusion was negligible. They indicated the swelling-breakdown to be controlled by the swelling capacity of the outer droplet (i.e. the oil layer resistance against breakdown) and that having higher lipophilic emulsifier leads to higher swelling capacities of W/O/W double emulsions (also observed by $[15,44]$ ). This was explained by the fact that increasing the surface of inner droplets, as a consequence of swelling, required an increasing amount of lipophilic surfactant. Similarly, Mezzenga et al. (2004) [46] stated that increasing the lipophilic surfactant fraction decreases the interfacial tension, which in turn decreases the Laplace pressure, leading to an increased swelling ratio. The overswelling-breakdown phenomenon was recently employed as a way to form hydrogel microfibers with precise length, where the swelling was induced via an alternating current electric field [47]. By investigating single drop-in-drop double emulsions Bahtz et al. (2015) [43] observed a lag stage (of about tens of minutes) at the beginning of storage, during which the swelling rate was very slow, as also reported by [11]. They indicated the lag duration to be higher for 
more viscous oil layers and lower for a higher inner phase fraction, while the osmotic pressure did not show any effect on the lag time duration [43].

In summary, the release of the encapsulated substance can generally be of different types: (a) Molecular diffusion of the encapsulated substance through the oil layer (without film rupture) which is generally slow if the encapsulated substance has a low solubility in the intermediate layer [11,13], (b) leakage of inner droplets due to breakage of the outer droplets by coalescence with the outer phase (only during preparation) $[5,6,23,30]$, (c) escape of inner droplets due to their Brownian motion (during storage), by coalescence to the external continuous phase through the surface of the outer droplets $[12,14]$; this phenomenon might be enhanced if there is a simple shear applied on the double emulsion, without necessarily breaking the outer droplets [28] (d) swelling of the inner (and so of the outer) droplets may enhance the escape of inner droplets, which is in this case called swelling-breakdown [11], e) extreme swelling may lead to overswelling-breakdown where a burst escape of inner droplets is observed causing breakage of the outer droplet.

It appears from the literature review that the preparation of double emulsions and their stability during storage are governed by a number of operating conditions that need further investigation. More precisely, the swelling and overswelling-breakdown phenomena need quantification. Also, correlations between the operating parameters and the properties just after preparation as well as during storage are required.

Objectives. The objective of this work is to investigate the effect of different operating conditions on the properties of the double emulsions during preparation and storage. During preparation, the breakage of the outer droplets and leakage of inner droplets are investigated. During storage, the escape and oveswelling-breakdown phenomena are 
investigated to determine their original causes. A specific attention is paid to investigate the full size distribution (DSD) of the inner and outer droplets. A number of key process parameters were varied, namely the fraction of internal water, the stirring rate in the second preparation step, the fraction of salt, and the fraction of primary emulsions. The first preparation step is done using ULTRA-TURRAX ${ }^{\circledR}$. The second emulsification step is done in a 1-L stirred vessel that is equipped with an in situ online video probe and a conductivity probe. The inner droplet size was measured right after the first preparation step. The outer droplet size and the conductivity of the double emulsions (which is indicative of the release

rate) were monitored during the second preparation step using both online and offline measurements, and during storage using offline measurements.

\section{Experimental}

\subsection{Materials}

W/O/W double emulsions are prepared using Mineral oil (Fisher ScientificTM), Span 80 (Alfa Aesar) as hydrophobic internal emulsifier, Tween 80 (Fisher ScientificTM) as hydrophilic external emulsifier, Sodium Chloride as tracer and Millipore water (resistivity $\approx 18.2$ $m \Omega . c m)$.

\subsection{Double emulsion preparation}

A two-step method was used to produce W/O/W double emulsions at room temperature. First, a primary emulsion was prepared by dispersing the $\mathrm{NaCl}$ aqueous solution in the oil phase - oil containing Span 80 - using an IKA T 25 digital ULTRA-TURRAX ${ }^{\circledR}$ at 12000 rpm for 4 min. Second, the double emulsion was produced by dispersing the primary emulsion in an 
external aqueous phase - water containing Tween 80 - in a 1-L vessel stirred at $300-500$ rpm for $70 \mathrm{~min}$ (Fig. 1). The fractions of these materials can be seen in Table 1.

\subsection{Conductivity measurements}

The conductivity measurement was performed using a CDM210 Conductivity Meter (MeterLab ${ }^{\circledR}$ ) during the second preparation step. The conductivity data allowed to predict the released amount of salt using a predetermined calibration curve, by dissolving different salt concentration in pure water. The samples were kept at controlled room temperature of $20^{\circ} \mathrm{C}$.

\subsection{Droplet size measurement}

The size of the inner droplets was measured right after the first preparation step by means of dynamic light scattering (Malvern Zetasizer Nano ZS ${ }^{\circledR}$ ). The samples were diluted approximately 1:1000 in mineral oil and the analysis was repeated twice.

The size of the outer droplets was measured by two techniques. First, an offline laser diffraction technique was employed (Malvern Mastersizer $3000^{\circledR}$ ). Samples were regularly taken during the second preparation step and during storage. The refractive index of the outer droplets was considered to be that of oil (i.e. 1.467), which represents the main component of these droplets, especially close to the surface. Second, an in situ video probe EZ Probe-D25 ${ }^{\circledR}$ was employed to take videos of the emulsion (Fig. 1), followed by image processing and reconstruction of the number density distribution. The probe was located 5 $\mathrm{cm}$ above the stirrer and close to the stirring shaft as suggested by [48]. The video probe has a CCD camera with a recording rate of 50 frames per second. Each video duration was $30 \mathrm{~s}$, from which 300 frames - of size $720 \times 576$ pixels - were selected. Image processing was then done offline using a MATLAB ${ }^{\circledR}$ program based on the circular Hough transform [48]. Besides 
these two quantitative methods, double emulsions were observed with an optical microscope to provide a visual analysis of inner and outer droplets (Leica C DM2000 LED).

\section{Results and discussions}

\subsection{Evidence of over swelling and overswelling-breakdown}

An example of the obtained inner droplet size distribution, measured by dynamic light scattering after the first preparation step is shown in Fig. 2. In general, inner droplets with a monomodal distribution around $1 \mu \mathrm{m}$ in mean diameter are obtained. After the production of the double emulsion (and during storage), quantitative measurement of the inner droplet size distribution represents a big challenge. Recent investigations involve the use of pulsed field gradient NMR technique (PFG-NMR) [8,49-54], and confocal laser scanning microscopy (CLSM) $[50,51,55,56]$, which are promising but they have different constraints and require mathematical treatments. In this work, a qualitative measurement is obtained by optical microscopy.

Regarding the outer droplets, two techniques were employed to measure the DSD. Indeed, while laser diffraction is nowadays a well-established method to measure the size of single emulsions, it is worthy to validate that the inner droplets do not impact the measurement of the outer droplets and eliminate possible effects of the dilution and pumping procedure in the Mastersizer (e.g. breakage or coalescence). Therefore, besides offline measurement using the master sizer, a video probe was inserted into the stirred vessel and the images were treated to predict the outer DSD. Fig. 3 shows an example of one 2-D image of the outer droplets taken in situ during the second emulsification step. The image processing treatment allows the detection of the outer droplets that are surrounded by circles, where 
most of them appear to be correctly detected. A comparison between the two techniques is shown in Fig. 4. In order to compare the results of the two methods, the size distributions were first converted into number densities then the real volume fractions were determined. It can be seen that the video probe has a limited capacity to detect very small droplets that are better captured by the master sizer. However, a fairly similar DSD is obtained by both methods. It can therefore be concluded that the inner emulsion does not influence the measurement in laser diffraction and that the double emulsion is stable during the measurement. Since the results from the Mastersizer are based on a bigger number of droplets and they give smoother curves, in the rest of the paper only these measurements are shown.

The DSD of the outer droplets were also qualitatively validated by optical microscopy. Besides, the microscopy represents the only available method in this work to investigate the evolution of the inner droplets after the production of the double emulsions. Fig. 5 shows few examples of microscopic images made during storage. At day 0 , right after the preparation of the double emulsion, both the inner and outer droplets can clearly be seen and their sizes are coherent with the measurements given in Figs $2-4$. At day 6 (Fig. 5B), it can be seen that the inner and outer droplets importantly swell. At day 9 (Fig. 5C), the droplets breakdown and decrease importantly in size. Full escape was calculated not to lead to this decrease in the size, which reveals the occurrence of breakage of the outer droplets. It appears that the outer droplets reach a maximum swelling capacity (here observed at day 6). This causes their breakdown (here observed at day 9), resulting in fast inner droplets escape to the external phase and outer droplet breakage. Fig. $5 \mathrm{C}$ shows that few inner droplets remain inside the outer droplets at day 9. These inner droplet did not overswell, certainly due to their initial smaller size and so higher Laplace pressure that counterbalances 
the osmotic pressure. They continue to release to the external phase via diffusion to the surface of the outer droplet and coalescence to the external phase. At day 50 all the outer droplets appear to be empty from the inner droplets (Fig. 5D, note that the zoom bare is reduced in this figure to $20 \mu \mathrm{m}$ to confirm that the outer droplets are empty).

Other than swelling, the increase in the outer droplet size during storage could be due to outer-outer droplet coalescence or Ostwald ripening. However, a single O/W emulsion $\left(\phi_{\text {Mineral oil }+ \text { Span } 80}=1 \%, \phi_{\text {Tween } 80}=1 \%\right.$ and $\left.\phi_{\text {Water }}=98 \%\right)$ was prepared and stored at similar conditions like the double emulsion and the increase in the size by coalescence or Oswald ripening was found to be negligible over 3 weeks. A comparison between this $\mathrm{O} / \mathrm{W}$ emulsion and a W/O/W double emulsion after one week of storage is shown in Fig. 6 . It can be seen that the initial sizes of both the single and double emulsion are comparable, and therefore they may be expected to undergo similar coalescence rates. After one week of storage, the droplet size of the O/W emulsion increased only very slightly due to droplet coalescence, while the outer droplet size of the W/O/W double emulsion increased importantly. This indicates that the main increase in the $\mathrm{W} / \mathrm{O} / \mathrm{W}$ double emulsion is due to swelling, which is driven by the osmotic pressure gradient caused by the presence of salt in the inner phase.

Fig. 7 shows an example of the evolution of the outer DSD over three weeks. Two stages can be distinguished from the figure: a) During the first week, the outer droplets get bigger in size due to swelling until reaching a maximum swelling (observed here at day 8 ), and b) during the following weeks, a decrease in their size is observed due to overswellingbreakdown. After three weeks, the final size of the outer droplets is smaller than their initial size. Such a decrease could not be explained by the escape of the internal phase alone. Indeed, the theoretical final mean outer droplet size was calculated, based on the initial size 
and the inner fraction, by assuming only escape (Fig. 8). It can be seen that the measured final size is smaller than the predicted one if only escape occurs. This confirms the occurrence of breakage of the outer droplets during the overswelling-breakdown phenomenon.

In the following sections, the effect of different key process parameters on the outer DSD and release rate, during preparation and storage, are investigated and discussed: the fraction of internal water, the stirring rate in the second preparation step, the fraction of salt, and the fraction of primary emulsion.

\subsection{Effect of the primary emulsion fraction ( $\phi_{\text {outer }}$ )}

The primary emulsion was prepared with $40 \%$ inner phase and $0.05 \%$ salt. Different amounts of this emulsion were then introduced in the stirred vessel under $400 \mathrm{rpm}$ for $70 \min ($ Table 1$)$.

During preparation, increasing the dispersed phase fraction may enhance droplet coalescence and reduce the breakage efficiency due to the damping effect of the energy dissipation [57]. Indeed, the energy dissipation rate $\varepsilon\left(\mathrm{W} \cdot \mathrm{kg}^{-1}\right)$ in a stirred tank can be calculated using the general power equation, $\varepsilon=\frac{N_{\mathrm{P}} \omega_{\mathrm{R}}^{3} D_{\mathrm{R}}^{5}}{V}$, where the power number of the employed three blade Mixel TT propeller is $N_{\mathrm{P}}=0.8, \omega_{\mathrm{R}}\left(\right.$ rev. $\left.\mathrm{s}^{-1}\right)$ is the stirring rate, $D_{\mathrm{R}}=$ $0.088 \mathrm{~m}$ is the impeller diameter and $V\left(\mathrm{~m}^{3}\right)$ is the volume of the material in the reactor. This gives $\varepsilon=1.25 \mathrm{~W} . \mathrm{kg}^{-1}$ with a mixing rate of $400 \mathrm{rpm}$. For a two-phase system, a dampening effect was highlighted where the effective energy dissipation rate becomes $\varepsilon_{\text {eff }}=\frac{\varepsilon}{\left(1+\phi_{\text {outer }}\right)^{3}}$ (with $\phi_{\text {outer }}$ the fraction of the outer phase) $[5,56]$. This gives $\varepsilon_{\text {eff }}=1.21$, $1.18,1.14$, and 1.11 for $\phi_{\text {outer }}=1,2,3$, and $4 \%$, respectively. Therefore, increasing the outer 
phase fraction from $1 \%$ to $4 \%$ slightly lowers the effective energy dissipation rate. From Fig. 9A, it can be seen that increasing $\phi_{\text {outer }}$ leads to slightly larger distributions and bigger droplets, except with $\phi_{\text {outer }}=4 \%$. However, the effect is not significant which can be explained by the fact that the emulsions are dilute as well as the use of a big amount of surfactant. A slight increase in the outer droplet size with increasing $\phi_{\text {outer }}$ from 2 to $7 \%$ was reported by [33]. Fig. 9B shows the released fraction estimated from the conductivity measurements. Similar release rates were obtained with most fractions, except the one with $\phi_{\text {outer }}=1 \%$ that led to a slightly higher release during preparation, indicating a slightly higher escape of inner droplets by the shear-induced breakage of the outer droplets $[2,5,6,30]$. A lower release (i.e., higher encapsulation efficiency) was reported when increasing $\phi_{\text {outer }}$ by [2] for more concentrated double emulsions (i.e., $\phi_{\text {outer }}=10-50 \%$ ). In the present system, the solubility of salt in the oil phase is low which leads to negligible release by molecular diffusion during preparation and storage. Thus, leakage due to breakage is the main cause of release $[5,23]$.

The samples were then stored at room temperature and the size and release rate were monitored during three weeks. A swelling ratio was calculated, as the ratio between the outer droplet mean diameter $\mathrm{d}_{43}$ during storage to the initial diameter obtained right after the preparation [15]. Fig. 9C shows that the swelling ratio reaches a maximum between one to two weeks then starts decreasing. The swelling ratio appears to be higher for higher primary emulsion fractions. Fig. 9D shows the release profile during storage. The presented release fraction is the part released during storage and is not cumulated with the released amount during preparation. At the beginning of storage, the release rate is low and is governed by the escape of inner droplets, due to their Brownian motion followed by inner droplet coalescence with the outer phase. This is in agreement with previous findings, 
where the release mechanism was found to be controlled by escape for an W/O/W double emulsion containing over 100 CMC of hydrophilic emulsifier with HLB < 30 (e.g. Tween 80) $[12,13]$. Then, a burst release is observed between one to two weeks, which corresponds to the time where the maximum swelling ratio is reached and overswelling-breakdown occurs. This phenomenon is caused when exceeding the swelling capacity of the double emulsion. This causes faster inner droplet escape and outer droplet breakage. Fig. 9D shows that the samples with lower swelling have higher release rates, which was also observed by [11].

Fig. 10 shows the initial, maximum swollen and final broken-down DSD of the outer droplets measured during storage for the different primary emulsion fractions. The full distribution gives supplementary information compared to the swelling ratio that is based on a mean diameter. It can be seen that the occurrence of the overswelling breakdown is slightly delayed when increasing the primary emulsion fraction. Namely, the DSD of the experiment with $\phi_{\text {outer }}=4 \%$ is bimodal at three weeks where part of the droplets is still at the maximum swelling capacity while another part has brokendown to small sizes.

\subsection{Effect of the salt fraction $\left(\phi_{\text {NaCl }}\right)$}

In this set, the fraction of salt in the internal phase was varied over the range of $0.05,0.14$, 0.19 and $0.24 \%$. The primary emulsions - consisting of $40 \%$ of the inner phase fraction were used to produce double emulsions at a fraction of $\phi_{\text {outer }}=1 \%$.

Fig. $11 \mathrm{~A}$ indicates that similar outer DSD are obtained with the different salt fractions except the one at the lowest salt fraction $\left(\phi_{\mathrm{NaCl}}=0.05 \%\right)$ that leads to the formation of much smaller outer droplets during the second preparation step. The presence of ions was found in the literature to play a complex role, thus affecting surfactant spreading and the rheology of the oil layer, which may explain this observation. This measurement is in line with the 
release profiles (Fig. 11B), where a higher release rate is observed with the salt fraction of $\phi_{\mathrm{NaCl}}=0.05 \%$. This may be due to the greater number of breakage events and higher surface area generated during the preparation of this experiment (that has smaller outer droplets), which leads to an enhanced escape of the inner droplets. During storage, the swelling ratio increased slightly when increasing the salt fraction, mainly at the maximum swelling capacity (Fig. 11C). In addition, the maximum swelling appeared faster for higher salt fractions, due to the increase in the osmotic gradient. The released fraction follows the pathway observed before where a slow release due to inner droplets escape first takes place, then a fast release is observed when overswelling-breakdown occurs (Fig. 11D). A lower release rate is observed when higher swelling occurs (i.e., for higher salt fractions).

Fig. 12 shows the full DSD of the outer droplets with the different salt fractions during storage. The samples with higher salt fractions (i.e., higher osmotic pressure) swell more, which is in line with previous works $[15,41,43]$.

\subsection{Effect of the inner phase fraction ( $\phi_{\text {inner }}$ )}

In this set of experiments, the salt fraction was kept at $0.05 \%$ while the internal phase fraction was varied over the range of $\phi_{\text {inner }}=10,20,30$ and $40 \%$ in the primary emulsion. The double emulsions were prepared using $1 \%$ of primary emulsion.

It can be seen that increasing $\phi_{\text {inner }}$ leads to an increase in the outer droplet size (Fig. 13A) and a reduced release fraction (Fig. 13B). Similar findings appear in the literature, where for higher $\phi_{\text {inner }}$ bigger droplets $[5,7,34,35]$ and higher encapsulation efficiencies were reported $[2,5,26]$, while a lower encapsulation efficiency was observed by $[22,35]$. The increase in the size and encapsulation efficiency can be explained by the viscosity change in outer droplets. Indeed, changing the internal water fraction changes the viscosity of the outer droplets 
( $\mu_{\text {outer }}$ ) following the viscosity model of dispersions, for instance the model of Vermeulen (1955) [58], $\mu_{\text {outer }}=\frac{\mu_{\text {oil }}}{1-\phi_{\text {inner }}}\left(1+1.5 \phi_{\text {inner }} \frac{\mu_{\text {inner }}}{\mu_{\text {inner }}+\mu_{\text {oil }}}\right)$. Considering mineral oil with viscosity, $\mu_{\mathrm{oil}}=55 \mathrm{mPa} . \mathrm{s}$, the viscosity of the outer phase calculated based on the model of Vermeulen gives $\mu_{\text {outer }}=61.11,68.75,78.57$, and $91.67 \mathrm{mPa} . \mathrm{s}$ for $\phi_{\text {inner }}=10,20,30$ and $40 \%$, respectively. Increasing the inner droplet fraction $\left(\phi_{\text {inner }}\right)$ thus increases the viscosity of the outer droplets. More viscous outer droplets resist the deformation and breakage during preparation, thus leading to lower inner droplet leakage $[5,59]$. During storage, Fig. $13 \mathrm{C}$ shows that the maximum swelling ratio is slightly lower for the sample with the highest inner fractions, so the highest viscous outer droplets, and a negligible effect for the other samples. Bahtz et al. [43] observed a negligible effect of $\phi_{\text {inner }}$ while Yan and Pal [15] observed a decrease in the swelling ratio when increasing the internal phase fraction. Fig. 13D shows a similar behavior of the release fraction for all inner fractions, except for the highest $\phi_{\text {inner }}$ where a higher release is observed. This confirms the previous observation of lower release for higher swelling.

Fig. 14 shows the full outer DSD of the experiments with different $\phi_{\text {inner. }}$. The overswelling breakdown occurs at about two weeks for all the inner fractions, and so viscosities. The peak of very small droplets created after overswelling-breakdown for the experiments with the lowest $\phi_{\text {inner }}(10$ and $20 \%$ ) reveals a greater number of breakup due to the lower viscosity of these experiments (Fig. 14A and 14B). This information is not clearly visible on the swelling ratio as the mean size used to calculate the swelling ratio may not be influenced by this peak, which highlights the importance of considering the full distribution for interpretation. 


\subsection{Effect of the stirring rate $\left(\omega_{R}\right)$}

The internal phase and salt fractions were respectively kept at $40 \%$ and $0.05 \%$ in the primary emulsion. Then, the double emulsions were prepared using $1 \%$ of primary emulsion fraction under different stirring rates: $\omega_{R}=300,350,400$ and $500 \mathrm{rpm}$.

The energy dissipation was calculated for the different stirring rates as explained above

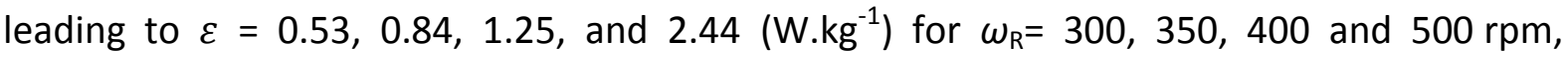
respectively. Therefore, increasing the stirring rate leads to a decrease in the outer droplet mean size and to a narrower distribution (Fig. 15A). Besides, an acceleration of inner droplet escape rate is observed (Fig. 15B). This is due to the greater number of breakage events taking place under higher stirring rates and the joint escape of inner droplets [5]. Indeed, the release is governed by the shear-induced breakage of the outer droplets during preparation $[5,6,30]$. Similar observations were highlighted in the literature regarding the effect of stirring rate (or energy) on the outer droplet diameter $[5,7,9,31,33,34]$ and the encapsulation efficiency $[5,22,25,27,29,30]$.

From Fig. 15C it can be seen that the swelling ratio is higher for the samples prepared with lower stirring rates, 300 and 350 rpm (i.e., bigger initial droples). This is confirmed on Fig. 16 showing the full DSDs, where big outer droplets can be observed for the samples prepared under 300 and $350 \mathrm{rpm}$. This is partly due to their initial bigger size combined to the fact that these double emulsions have a higher initial salt fraction (i.e., lower release during preparation). The overswelling-breakdown of the outer droplets occurs at around 2 weeks for all the samples showing that the initial size of the outer droplets does not have a direct effect on the oil layer rheology. As a consequence, the released fractions are all identical (Fig. 15D). 


\section{Conclusions}

The evolution of the outer droplet size and release rate in dilute double emulsions during the second step of preparation and storage was studied by varying different key process parameters.

During preparation, the breakage governs the size of the outer droplets and the release rate (leakage). It was found that varying the primary emulsion fractions from 1 to $4 \%$ did not affect the size of outer droplets nor the release. Increasing the salt fraction from 0.05 to $0.14 \%$ led to more swelling and lower release. Increasing the internal phase fraction generated more viscous outer droplets with higher resistivity against breakage leading to bigger outer droplets and lower release rates. Increasing the stirring rate - the main cause of breakage - allowed to create smaller sizes and led to higher release fractions.

During the storage time, two periods can be distinguished. A first period, of about one to two weeks, corresponds to swelling of the inner, and so of the outer droplets, and escape of inner droplets by Brownian motion through their coalescence with the external phase through the surface of the outer droplet. The second period appears once the maximum swelling capacity is reached and overswelling-breakdown occurs. In this case, a faster inner droplet escape is observed. Moreover, it appears that the outer droplet size is smaller than the initial one (before swelling) and smaller than the theoretical size when assuming full inner droplet escape. This indicates that the overswelling-breakdown causes breakage of the outer droplets, not only full release of inner droplets. Investigating the effect of the different key process parameters indicated that higher primary emulsion fractions resulted in more swelling and so less release and delayed breakdown. The samples with high salt fractions reached very high swelling ratios. Increasing the internal phase fractions slightly 
lowered the maximum swelling ratio, due to the increased overall viscosity of the outer droplets. Bigger initial outer droplets, prepared with lower stirring rates, swelled more due to their higher initial salt fractions (i.e. lower release during preparation).

It appears that the system having $40 \%$ inner and $4 \%$ outer phase fractions, $0.14 \%$ salt fraction, and $400 \mathrm{rpm}$ stirring rate would lead to a final product having smaller outer droplets and lower release, which represent the main indicators of quality products. Besides, this system proves a longer delay in undesired overswelling-breakdown while keeping salt and stirring rate at low value.

This detailed study provides an important insight in the preparation of double emulsions and on the overswelling-breakdown phenomenon during storage. The results can be useful for formulation of more stable quality products.

\section{Acknowledgements}

This work was funded by ModLife ITN. Grant agreement number 675251. 


\section{References}

[1] N.N. Li, Separating hydrocarbons with liquid membranes, Google Patents, 1968.

[2] S. Matsumoto, Y. Kita, D. Yonezawa, An attempt at preparing water-in-oil-in-water multiple-phase emulsions, J. Colloid Interface Sci. 57 (1976) 353-361. doi:10.1016/0021-9797(76)90210-1.

[3] G. Muschiolik, I. Scherze, P. Preissler, J. Weiß, A. Knoth, A. Fechner, Multiple Emulsions - Preparation and Stability, in: 13th World Congr. Food Sci. Technol., EDP Sciences, Nantes, France, 2006. doi:10.1051/IUFoST:20060043.

[4] L. Zeng, Y. Zhang, C. Bukirwa, W. Li, Y. Yang, Study of mean diameter and drop size distribution of emulsion drops in a modified rotating disc contactor for an emulsion liquid membrane system, RSC Adv. 5 (2015) 89959-89970. doi:10.1039/C5RA16267J.

[5] B. Khadem, N. Sheibat-Othman, Modeling of double emulsions using population balance equations, Chem. Eng. J. 366 (2019) 587-597. doi:10.1016/j.cej.2019.02.092.

[6] A.J. Shere, H.M. Cheung, MODELING OF LEAKAGE IN LIQUID SURFACTANT MEMBRANE SYSTEMS, Chem. Eng. Commun. 68 (1988) 143-164. doi:10.1080/00986448808940403.

[7] T. Ohtake, T. Hano, K. Takagi, F. Nakashio, Analysis of water entrainment into dispersed W/O emulsion drops., J. Chem. Eng. Jpn. 21 (1988) 272-276. doi:10.1252/jcej.21.272.

[8] X. Guan, K. Hailu, G. Guthausen, F. Wolf, R. Bernewitz, H.P. Schuchmann, PFG-NMR on W1/O/W2-emulsions: Evidence for molecular exchange between water phases, Eur. J. Lipid Sci. Technol. 112 (2010) 828-837. doi:10.1002/ejlt.201000022.

[9] A. Schuch, J. Wrenger, H.P. Schuchmann, Production of W/O/W double emulsions. Part II: Influence of emulsification device on release of water by coalescence, Colloids Surf. Physicochem. Eng. Asp. 461 (2014) 344-351. doi:10.1016/j.colsurfa.2013.11.044.

[10] S. Mataumoto, W.W. Kang, FORMATION AND APPLICATIONS OF MULTIPLE EMULSIONS, J. Dispers. Sci. Technol. 10 (1989) 455-482. doi:10.1080/01932698908943184.

[11] N. Jager-Lezer, I. Terrisse, F. Bruneau, S. Tokgoz, L. Ferreira, D. Clausse, M. Seiller, J.-L. Grossiord, Influence of lipophilic surfactant on the release kinetics of water-soluble molecules entrapped in a W/O/W multiple emulsion, J. Controlled Release. 45 (1997) 1-13. doi:10.1016/S0168-3659(96)01507-6.

[12] K. Pays, J. Giermanska-Kahn, B. Pouligny, J. Bibette, F. Leal-Calderon, Coalescence in Surfactant-Stabilized Double Emulsions, Langmuir. 17 (2001) 7758-7769. doi:10.1021/la010735x.

[13] K. Pays, Double emulsions: how does release occur?, J. Controlled Release. 79 (2002) 193-205. doi:10.1016/S0168-3659(01)00535-1.

[14] M. Chávez-Páez, C.M. Quezada, L. Ibarra-Bracamontes, H.O. González-Ochoa, J.L. Arauz-Lara, Coalescence in Double Emulsions, Langmuir. 28 (2012) 5934-5939. doi:10.1021/la205144g.

[15] J. Yan, R. Pal, Osmotic swelling behavior of globules of W/O/W emulsion liquid membranes, J. Membr. Sci. 190 (2001) 79-91.

[16] S.S. Davis, A.S. Burbage, The Particle Size Analysis of Multiple Emulsions (Water-in-Oilin-Water), in: Part. Size Anal. Proc. Conf. Organ. Anal. Div. Chem. Soc. Held Univ. Salford 12-15 Sept. 1977, Heyden \& Son, 1978: p. 395.

[17] D. Whitehill, A.T. Florence, Mechanisms of instability in W/O/W multiple emulsions, J. Pharm. Pharmacol. 31 (1979) 3P-3P. 
[18] A.T. Florence, D. Whitehill, The formulation and stability of multiple emulsions, Int. J. Pharm. 11 (1982) 277-308.

[19] A.. Florence, D. Whitehill, Some features of breakdown in water-in-oil-in-water multiple emulsions, J. Colloid Interface Sci. 79 (1981) 243-256. doi:10.1016/00219797(81)90066-7.

[20] S. Geiger, S. Tokgoz, A. Fructus, N. Jager-Lezer, M. Seiller, C. Lacombe, J.-L. Grossiord, Kinetics of swelling-breakdown of a W/O/W multiple emulsion: possible mechanisms for the lipophilic surfactant effect, J. Controlled Release. 52 (1998) 99-107. doi:10.1016/S0168-3659(97)00202-2.

[21] S. Raynal, J.L. Grossiord, M. Seiller, D. Clausse, A topical W/O/W multiple emulsion containing several active substances: formulation, characterization and study of release, J. Controlled Release. 26 (1993) 129-140. doi:10.1016/0168-3659(93)90112-I.

[22] T.S.H. Leong, M. Zhou, N. Kukan, M. Ashokkumar, G.J.O. Martin, Preparation of waterin-oil-in-water emulsions by low frequency ultrasound using skim milk and sunflower oil, Food Hydrocoll. 63 (2017) 685-695. doi:10.1016/j.foodhyd.2016.10.017.

[23] B. Khadem, N. Sheibat-Othman, Theoretical and experimental investigations of double emulsion preparation by ultrasonication, Ind. Eng. Chem. Res. (2019). doi:10.1021/acs.iecr.9b00556.

[24] S. Matsumoto, Y. Ueda, Y. Kita, D. Yonezawa, Preparation of Water-in-Olive Oil-inWater Multiple-phase Emulsions in an Eatable Form, Agric. Biol. Chem. 42 (1978) 739743. doi:10.1080/00021369.1978.10863054.

[25] E. Dickinson, Double Emulsions Stabilized by Food Biopolymers, Food Biophys. 6 (2011) 1-11. doi:10.1007/s11483-010-9188-6.

[26] A. Cárdenas, E. Castro, Breaking of multiple emulsions under osmotic pressure and the effect of W1/O relation, Interciencia. 28 (2003) 534-538.

[27] T.P. Martin, G.A. Davies, The extraction of copper from dilute aqueous solutions using a liquid membrane process, Hydrometallurgy. 2 (1977) 315-334. doi:10.1016/0304386X(77)90040-8.

[28] J.. Klahn, J.J.. Janssen, G.E.. Vaessen, R. de Swart, W.G.. Agterof, On the escape process during phase inversion of an emulsion, Colloids Surf. Physicochem. Eng. Asp. 210 (2002) 167-181. doi:10.1016/S0927-7757(02)00376-X.

[29] H. Okochi, M. Nakano, Preparation and evaluation of w/o/w type emulsions containing vancomycin, Adv. Drug Deliv. Rev. 45 (2000) 5-26. doi:10.1016/S0169-409X(00)000971.

[30] S. Okazaki, M. Imai, M. Shimizu, Leakage Suppressing Oe W/O Emulsion Using High Viscous Solvent, in: Process Metall., Elsevier, 1992: pp. 1487-1492. https://linkinghub.elsevier.com/retrieve/pii/B9780444886774500647 (accessed October 30, 2018).

[31] V. Muguet, M. Seiller, G. Barratt, D. Clausse, J.. Marty, J.. Grossiord, W/O/W Multiple Emulsions Submitted to a Linear Shear Flow: Correlation between Fragmentation and Release, J. Colloid Interface Sci. 218 (1999) 335-337. doi:10.1006/jcis.1999.6374.

[32] K. Lindenstruth, B.W. Müller, W/O/W multiple emulsions with diclofenac sodium, Eur. J. Pharm. Biopharm. 58 (2004) 621-627. doi:10.1016/j.ejpb.2004.04.003.

[33] T. Gallego-Lizon, E.S. Pérez de Ortiz, Drop Sizes in Liquid Membrane Dispersions, Ind. Eng. Chem. Res. 39 (2000) 5020-5028. doi:10.1021/ie000016y.

[34] A. Sharma, A.N. Goswami, B.S. Rawat, Drop size prediction in liquid membrane systems, J. Membr. Sci. 60 (1991) 261-274. doi:10.1016/S0376-7388(00)81539-2. 
[35] A. Schuch, P. Deiters, J. Henne, K. Köhler, H.P. Schuchmann, Production of W/O/W (water-in-oil-in-water) multiple emulsions: droplet breakup and release of water, J. Colloid Interface Sci. 402 (2013) 157-164. doi:10.1016/j.jcis.2013.03.066.

[36] M. Frenkel, R. Shwartz, N. Garti, Multiple emulsions, J. Colloid Interface Sci. 94 (1983) 174-178. doi:10.1016/0021-9797(83)90247-3.

[37] T. Schmidts, D. Dobler, C. Nissing, F. Runkel, Influence of hydrophilic surfactants on the properties of multiple W/O/W emulsions, J. Colloid Interface Sci. 338 (2009) 184-192. doi:10.1016/j.jcis.2009.06.033.

[38] T. Schmidts, D. Dobler, P. Schlupp, C. Nissing, H. Garn, F. Runkel, Development of multiple W/O/W emulsions as dermal carrier system for oligonucleotides: Effect of additives on emulsion stability, Int. J. Pharm. 398 (2010) 107-113. doi:10.1016/j.ijpharm.2010.07.037.

[39] T. Hino, Y. Kawashima, S. Shimabayashi, Basic study for stabilization of w/o/w emulsion and its application to transcatheter arterial embolization therapy, Adv. Drug Deliv. Rev. 45 (2000) 27-45. doi:10.1016/S0169-409X(00)00098-3.

[40] M.-F. Ficheux, L. Bonakdar, F. Leal-Calderon, J. Bibette, Some Stability Criteria for Double Emulsions, Langmuir. 14 (1998) 2702-2706. doi:10.1021/la971271z.

[41] S. Matsumoto, M. Kohda, The viscosity of W/O/W emulsions: an attempt to estimate the water permeation coefficient of the oil layer from the viscosity changes in diluted systems on aging under osmotic pressure gradients, J. Colloid Interface Sci. 73 (1980) 13-20.

[42] S. Matsumoto, T. Inoue, M. Kohda, K. Ikura, Water permeability of oil layers in W/O/W emulsions under osmotic pressure gradients, J. Colloid Interface Sci. 77 (1980) 555563.

[43] J. Bahtz, D.Z. Gunes, E. Hughes, L. Pokorny, F. Riesch, A. Syrbe, P. Fischer, E.J. Windhab, Decoupling of Mass Transport Mechanisms in the Stagewise Swelling of Multiple Emulsions, Langmuir. 31 (2015) 5265-5273. doi:10.1021/acs.langmuir.5b01138.

[44] N. Garti, S. Magdassi, D. Whitehill, Transfer phenomena across the oil phase in wateroil-water multiple emulsions evaluated by Coulter counter: 1 . Effect of emulsifier 1 on water permeability, J. Colloid Interface Sci. 104 (1985) 587-591.

[45] I. Terrisse, M. Seiller, J.L. Grossiord, A. Magnet, C. Le Hen-Ferrenbach, Application of rheological analysis to W/O/W multiple emulsions: Effect of the incorporation of a coemulsifier, Colloids Surf. Physicochem. Eng. Asp. 91 (1994) 121-128. doi:10.1016/0927-7757(94)02790-0.

[46] R. Mezzenga, B.M. Folmer, E. Hughes, Design of Double Emulsions by Osmotic Pressure Tailoring, Langmuir. 20 (2004) 3574-3582. doi:10.1021/la036396k.

[47] X. Deng, Y. Ren, L. Hou, W. Liu, Y. Jia, H. Jiang, Electric Field-Induced Cutting of Hydrogel Microfibers with Precise Length Control for Micromotors and Building Blocks, ACS Appl. Mater. Interfaces. 10 (2018) 40228-40237. doi:10.1021/acsami.8b12597.

[48] A. Khalil, F. Puel, Y. Chevalier, J.-M. Galvan, A. Rivoire, J.-P. Klein, Study of droplet size distribution during an emulsification process using in situ video probe coupled with an automatic image analysis, Chem. Eng. J. 165 (2010) 946-957. doi:10.1016/j.cej.2010.10.031.

[49] R. Bernewitz, E. Caro, D. Topgaard, H.P. Schuchmann, G. Guthausen, Double Emulsion Character with PFG-NMR- Methods: WOW and OWO, in: F. Capozzi, L. Laghi, P.S. Belton (Eds.), Spec. Publ., Royal Society of Chemistry, Cambridge, 2015: pp. 120-126. http://ebook.rsc.org/?DOI=10.1039/9781782622741-00120 (accessed June 27, 2016). 
[50] R. Bernewitz, U.S. Schmidt, H.P. Schuchmann, G. Guthausen, Structure of and diffusion in O/W/O double emulsions by CLSM and NMR-comparison with W/O/W, Colloids Surf. Physicochem. Eng. Asp. 458 (2014) 10-18. doi:10.1016/j.colsurfa.2014.01.002.

[51] R. Bernewitz, F. Dalitz, K. Köhler, H.P. Schuchmann, G. Guthausen, Characterisation of multiple emulsions by NMR spectroscopy and diffusometry, Microporous Mesoporous Mater. 178 (2013) 69-73. doi:10.1016/j.micromeso.2013.02.049.

[52] J.P. Hindmarsh, J. Su, J. Flanagan, H. Singh, PFG-NMR Analysis of Intercompartment Exchange and Inner Droplet Size Distribution of W/O/W Emulsions, Langmuir. 21 (2005) 9076-9084. doi:10.1021/la051626b.

[53] I. Lönnqvist, B. Håkansson, B. Balinov, O. Söderman, NMR Self-Diffusion Studies of the Water and the Oil Components in a W/O/W Emulsion, J. Colloid Interface Sci. 192 (1997) 66-73. doi:10.1006/jcis.1997.4966.

[54] L. Vermeir, P. Sabatino, M. Balcaen, A. Declerck, K. Dewettinck, J.C. Martins, G. Guthausen, P. Van der Meeren, Effect of molecular exchange on water droplet size analysis as determined by diffusion NMR: The W/O/W double emulsion case, J. Colloid Interface Sci. 475 (2016) 57-65. doi:10.1016/j.jcis.2016.04.029.

[55] A. Lamprecht, U. Schäfer, C.-M. Lehr, Structural analysis of microparticles by confocal laser scanning microscopy, AAPS PharmSciTech. 1 (2000) 10-19. doi:10.1208/pt010317.

[56] S. Schuster, R. Bernewitz, G. Guthausen, J. Zapp, A.M. Greiner, K. Köhler, H.P. Schuchmann, Analysis of W1/O/W2 double emulsions with CLSM: Statistical image processing for droplet size distribution, Chem. Eng. Sci. 81 (2012) 84-90. doi:10.1016/j.ces.2012.06.059.

[57] C.A. Coulaloglou, L.L. Tavlarides, Description of interaction processes in agitated liquidliquid dispersions, Chem. Eng. Sci. 32 (1977) 1289-1297. doi:10.1016/00092509(77)85023-9.

[58] Theodore Vermeulen, Interfacial area in liquid-liquid and gas-liquid agitation, Chem. Eng. Progr. 51 (1955) 85F-94F.

[59] P. Julian Becker, F. Puel, H.A. Jakobsen, N. Sheibat-Othman, Development of an improved breakage kernel for high dispersed viscosity phase emulsification, Chem. Eng. Sci. 109 (2014) 326-338. doi:10.1016/j.ces.2014.02.008. 
Table 1. Experimental conditions

\begin{tabular}{|c|c|c|c|}
\hline \multicolumn{4}{|l|}{ First preparation step } \\
\hline Fractions (wt. \%) & & $\begin{array}{l}\text { Opera } \\
\text { param }\end{array}$ & $\begin{array}{l}\text { ting } \\
\text { eters }\end{array}$ \\
\hline Water Mineral oil $\mathrm{NaCl}$ & Span 80 & $\begin{array}{l}t_{1} \\
(\min )\end{array}$ & $\begin{array}{l}\omega_{\mathrm{RS}} \\
(\mathrm{rpm})\end{array}$ \\
\hline
\end{tabular}

Set 1:

$\phi_{\text {outer }}$

$40 \quad 50$

$0.05 \quad 9.95$

4

12000

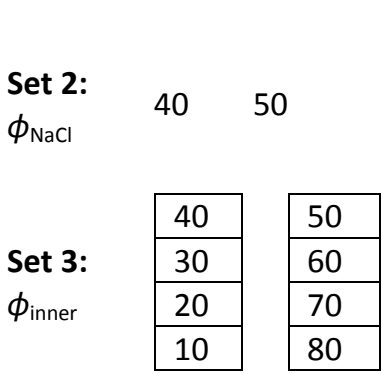

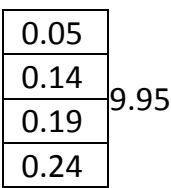

$0.05 \quad 9.95$

4

12000

1

98

1

$70 \quad 400$

\begin{tabular}{|l|}
\hline 1 \\
\hline 2 \\
\hline 3 \\
\hline 4 \\
\hline 9
\end{tabular} \mid \begin{tabular}{|l|}
\hline 98 \\
\hline 96 \\
\hline 95 \\
\hline
\end{tabular}

Operating parameters

$t_{2} \quad \omega_{\mathrm{B}}$

(min) $(\mathrm{rpm})$

$70 \quad 400$

$4 \quad 12000$

1

$98 \quad 1$

$70 \quad 400$

Set 4:

$\omega_{\mathrm{R}}$

$40 \quad 50$

$0.05 \quad 9.95$

4

12000

1

$98 \quad 1$

\begin{tabular}{|l|l|}
\hline \multirow{1}{*}{70} & 300 \\
\cline { 2 - 2 } & 350 \\
\hline & 400 \\
\hline & 500 \\
\hline
\end{tabular}

Symbols: $\phi_{\text {outer }}$ : Outer phase fraction, $\phi_{\mathrm{NaCl}}$ : Salt fraction, $\phi_{\text {inner }}$ : Inner phase fraction, $\omega_{\mathrm{R}}$ : stirring rate in the vessel, and $\omega_{\mathrm{RS}}$ : stirring rate by the Ultra-Turrax. The total mass in the second step is $1 \mathrm{~kg}$. 


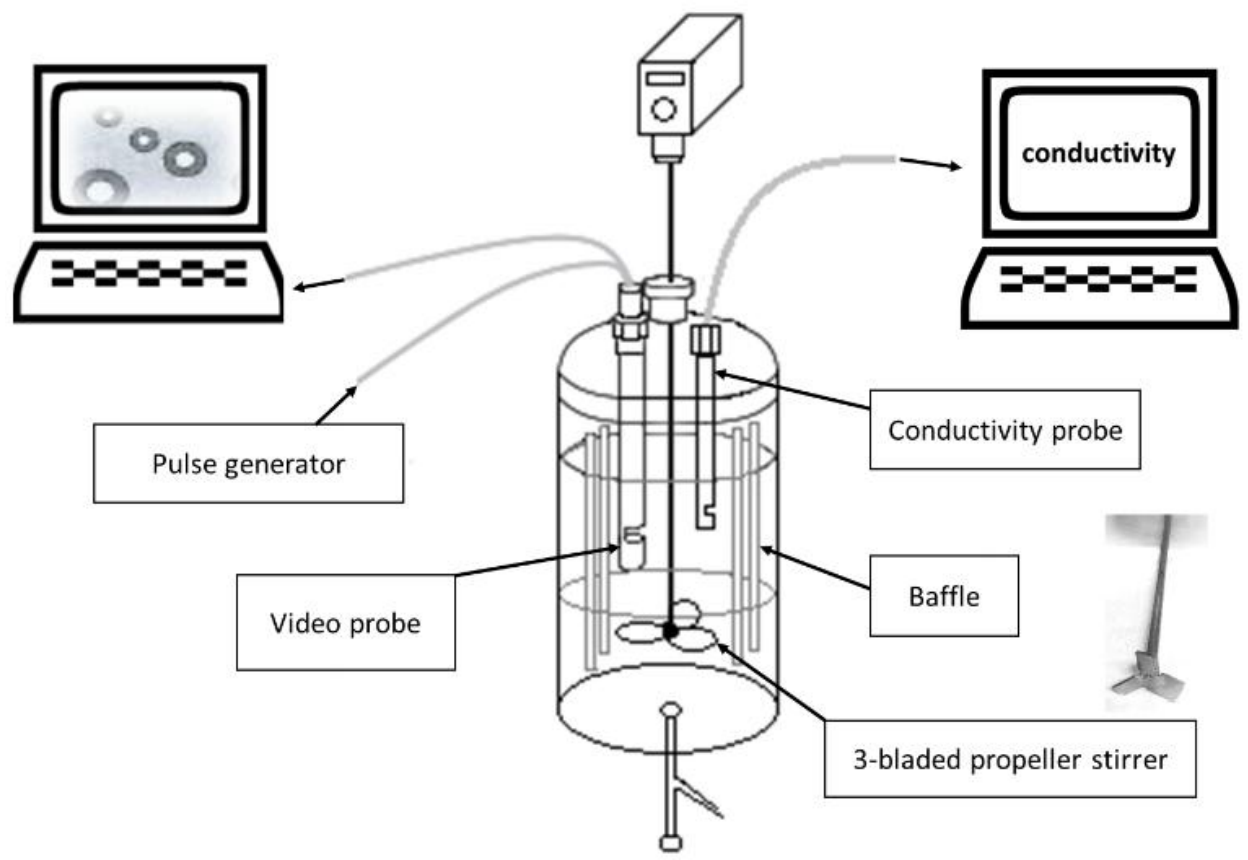

Fig. 1. Set-up for the second step of double emulsion preparation in a 1-L reactor. 


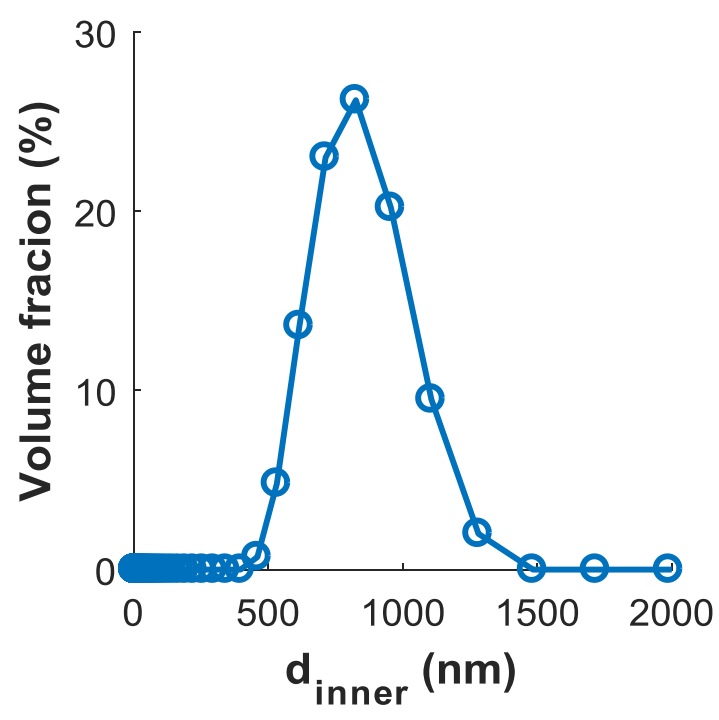

Fig. 2. A typical size distribution of inner droplets (i.e., the primary $W / O$ emulsion), prepared with $\omega_{R S}=12000 \mathrm{rpm}, \phi_{\text {inner }}=40 \%$ and $\phi_{\mathrm{NaCl}}=0.05 \%$. 


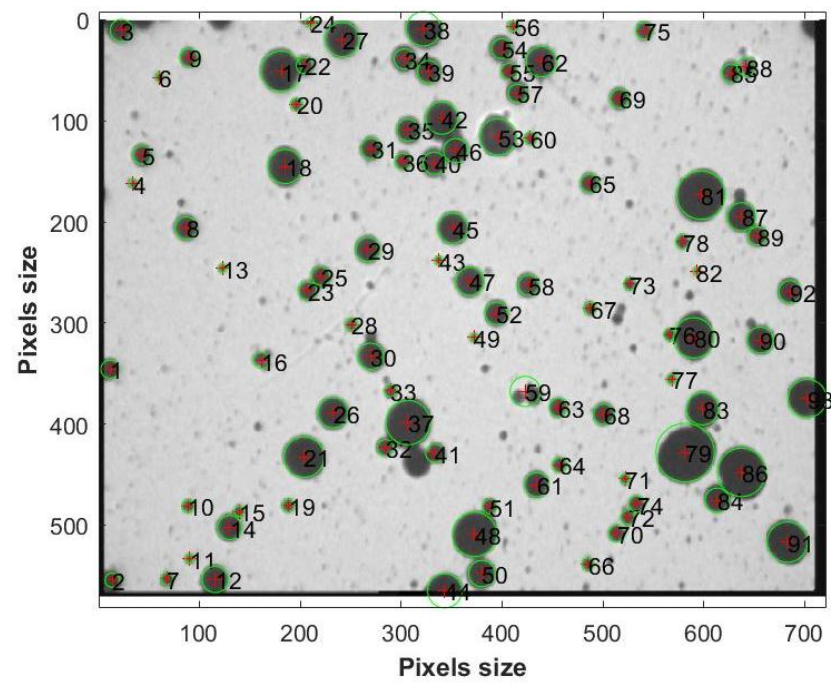

Fig. 3. An image extracted from the video probe measurements with the outer droplets detected by image processing, for the sample prepared with $\phi_{\text {inner }}=40 \%, \phi_{\mathrm{NaCl}}=0.05 \%$, $\phi_{\text {outer }}=1 \%$, and $\omega_{R}=300 \mathrm{rpm}$. 


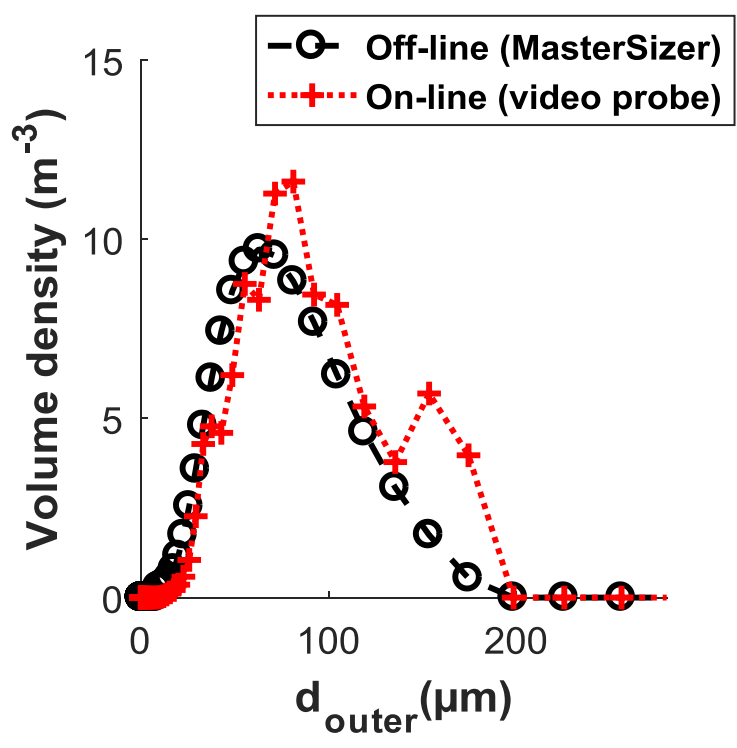

Fig. 4. A comparison of the outer droplet size distribution measured by MasterSizer and the video probe, for the sample prepared with $\phi_{\text {inner }}=40 \%, \phi_{\mathrm{NaCl}}=0.05 \%, \phi_{\text {outer }}=1 \%$, and $\omega_{R}=$ 300 rpm. 

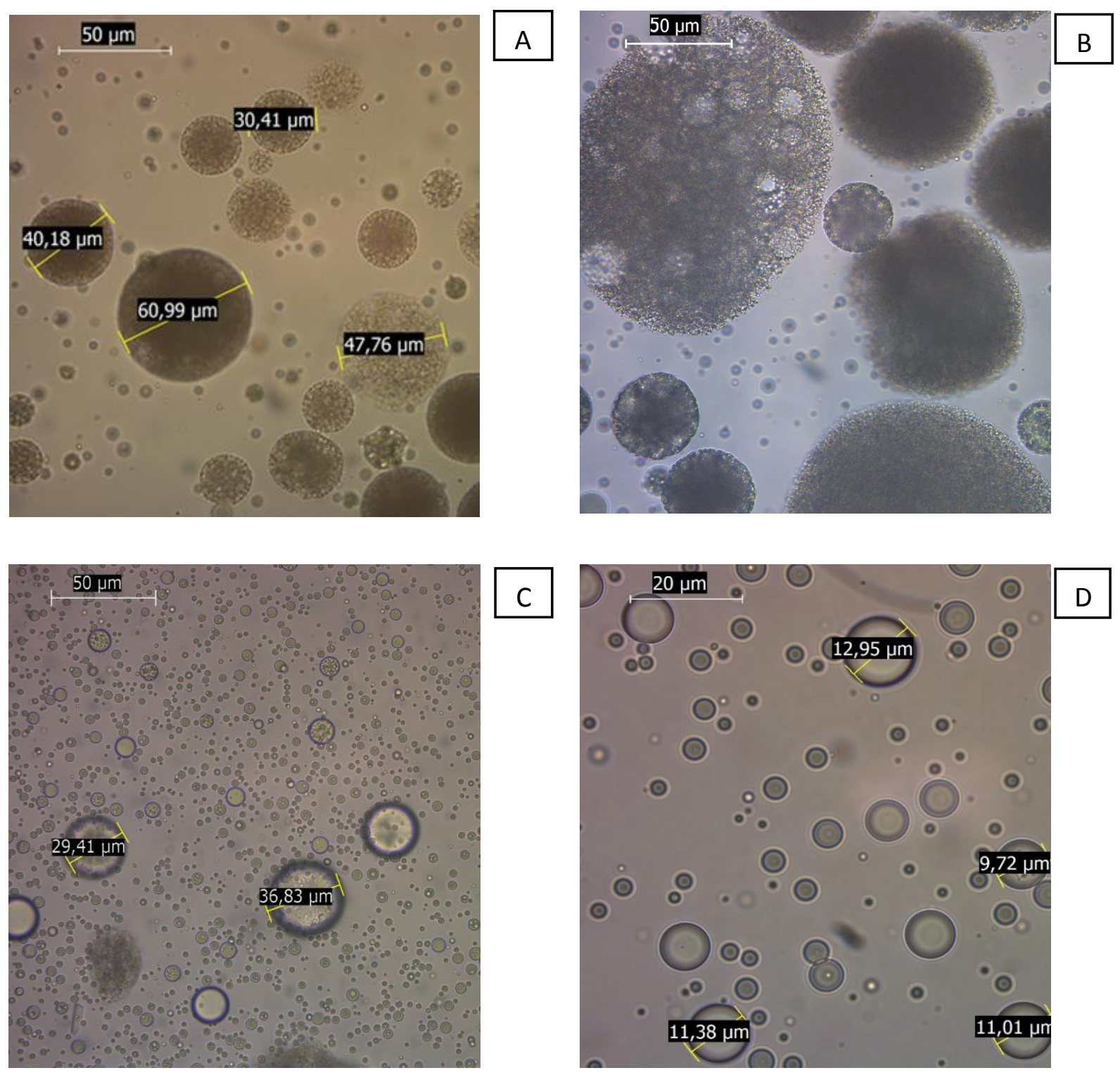

Fig. 5. Optical microscopic images, for the sample prepared with $\phi_{\text {inner }}=40 \%, \phi_{\text {outer }}=1 \%$, and $\omega_{R}=400$ rpm: A) Right after preparation (Day 0), B) Day 6, C) Day 9, and D) Day 50. 


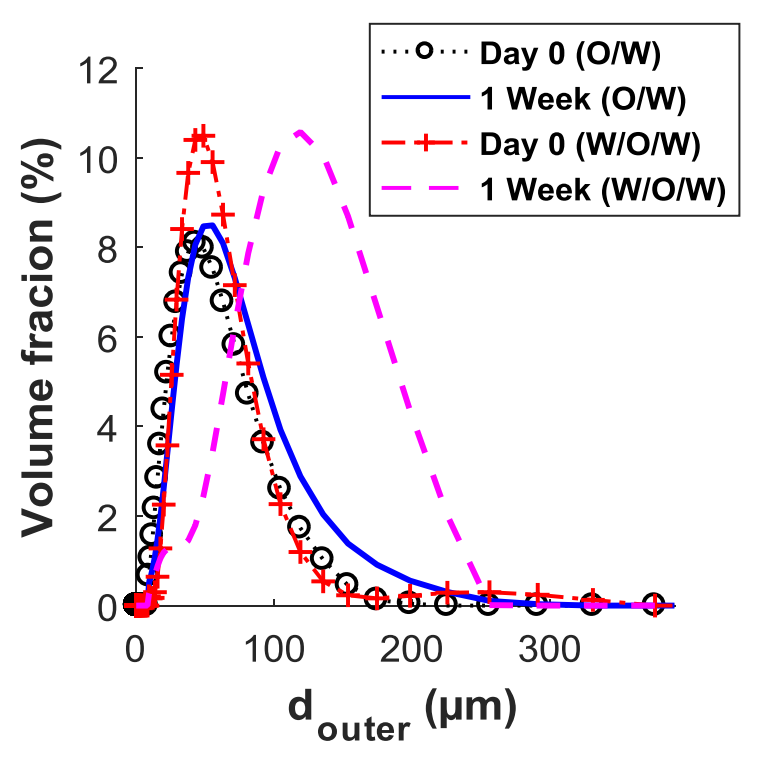

Fig. 6. Comparison between the DSD of a single O/W emulsion and the outer DSD of W/O/W double emulsion during one week of storage. $O / W$ is prepared with $\phi_{\text {oil phase }}=1 \%$ and $\omega_{R}=$ $400 \mathrm{rpm} . \mathrm{W} / \mathrm{O} / \mathrm{W}$ is prepared with $\phi_{\text {inner }}=40 \%, \phi_{\mathrm{NaCl}}=0.05 \%, \phi_{\text {outer }}=1 \%$ and $\omega_{R}=400 \mathrm{rpm}$ 


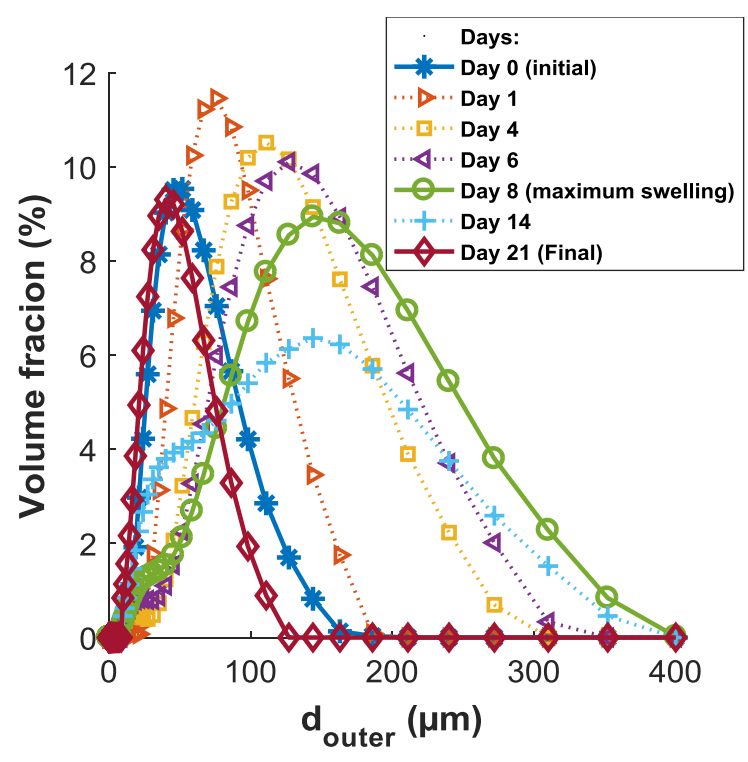

Fig. 7. Evolution of the outer droplet size distribution for the sample prepared with $\phi_{\text {inner }}=$ $40 \%, \phi_{\mathrm{NaCl}}=0.05 \%, \phi_{\text {outer }}=2 \%$ and $\omega_{R}=400 \mathrm{rpm}$. 


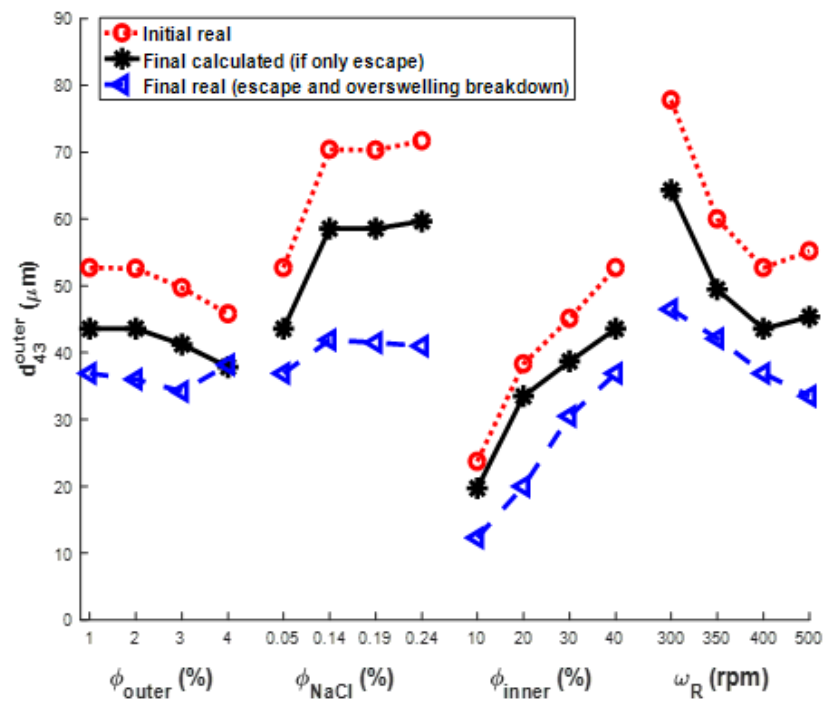

Fig. 8. Mean outer droplets diameter: Real initial $d_{43}$, real final $d_{43}$ and final predicted $d_{43}$ calculated assuming full escape (initial outer droplet volume - inner droplets volume). 

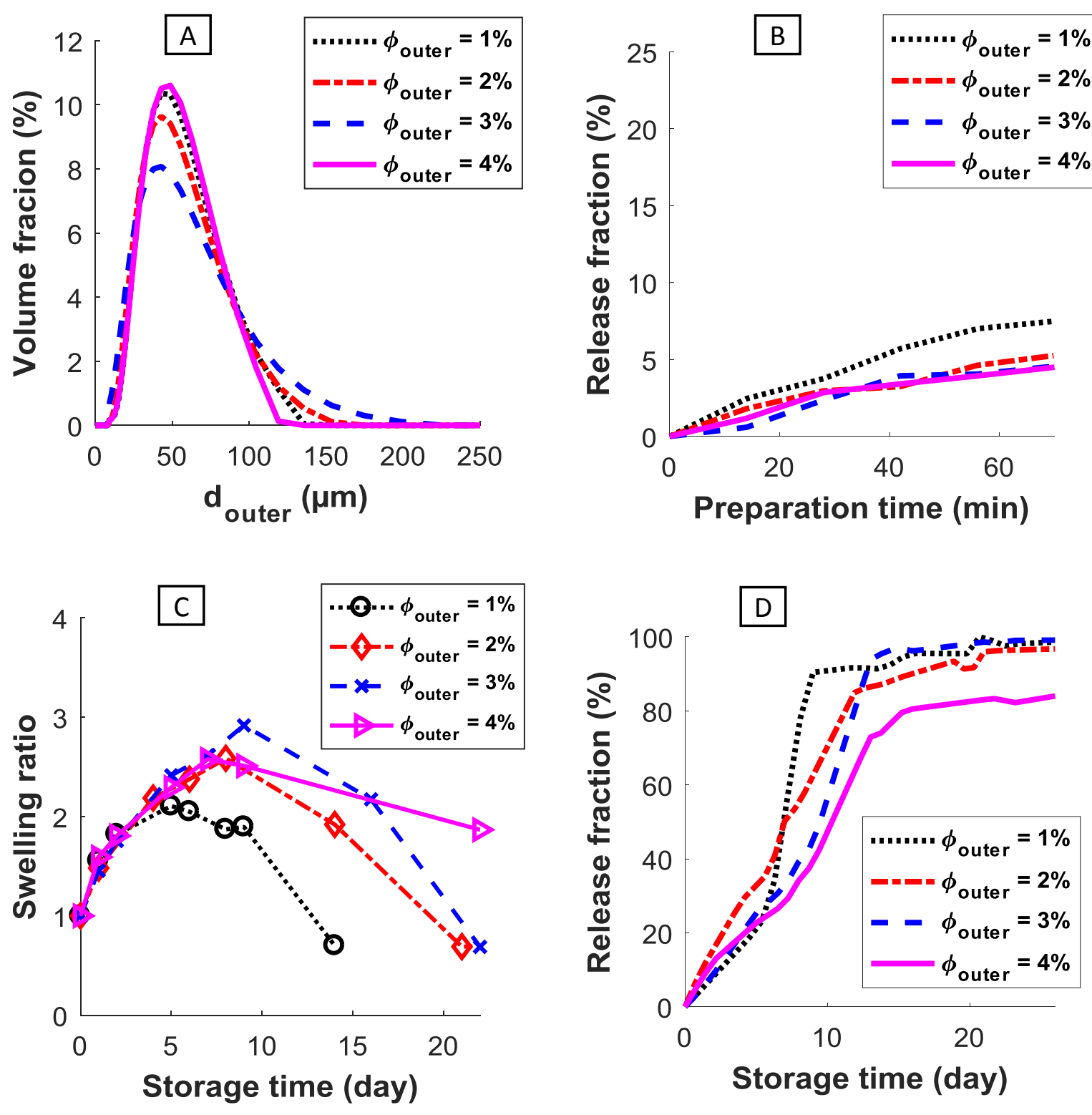

Fig. 9. Effect of the fraction of primary emulsion ( $\left.\phi_{\text {outer }}\right)$ on the: $\left.A\right)$ droplet size distribution during preparation, $B$ ) release during preparation, C) swelling ratio during storage (i.e. the ratio of the mean diameter $d_{43}$ after and before swelling), and D) released fraction. 

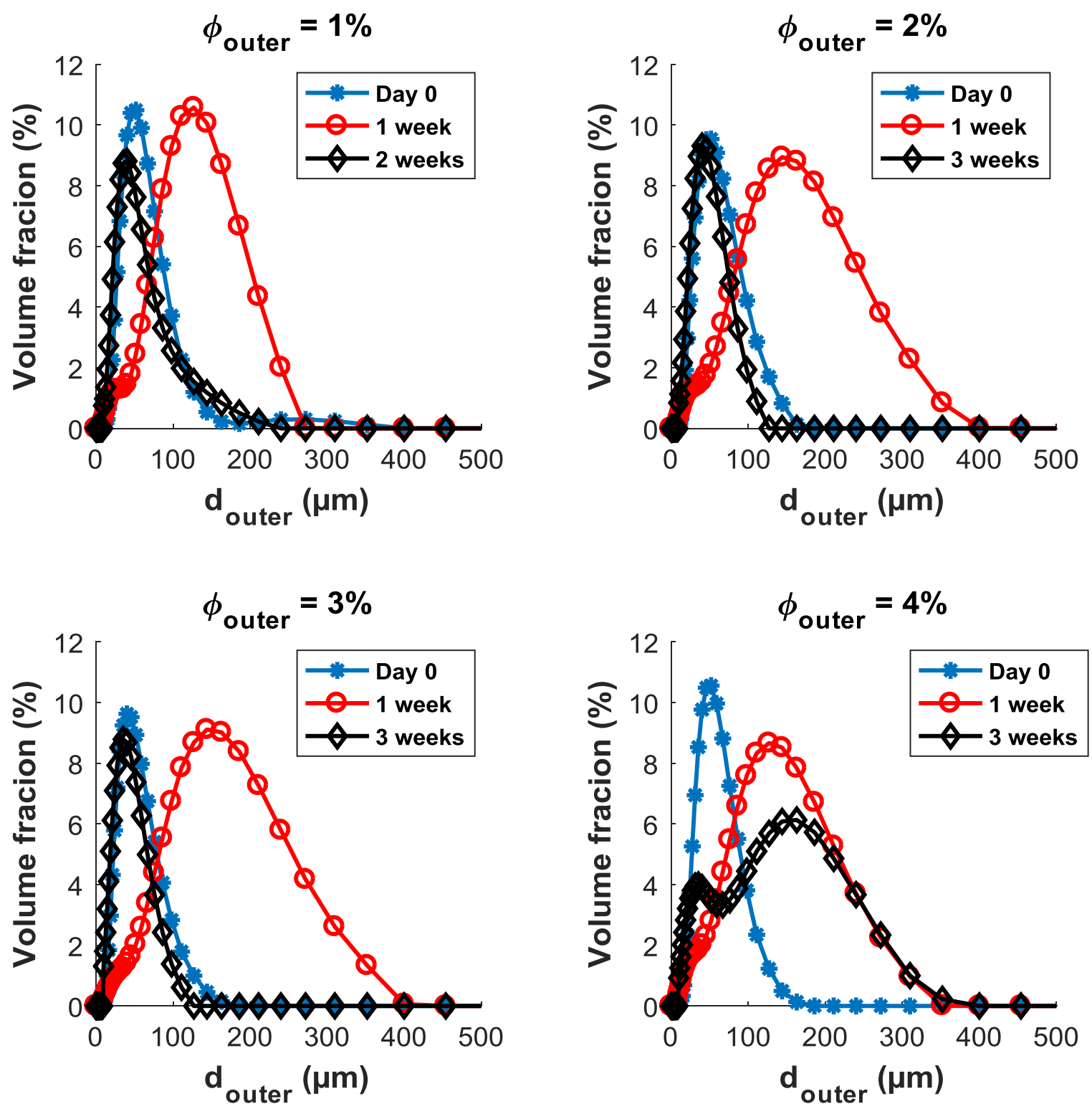

Fig. 10. Effect of primary emulsion fraction ( $\left.\phi_{\text {outer }}\right)$ on the droplet size distribution during storage. 

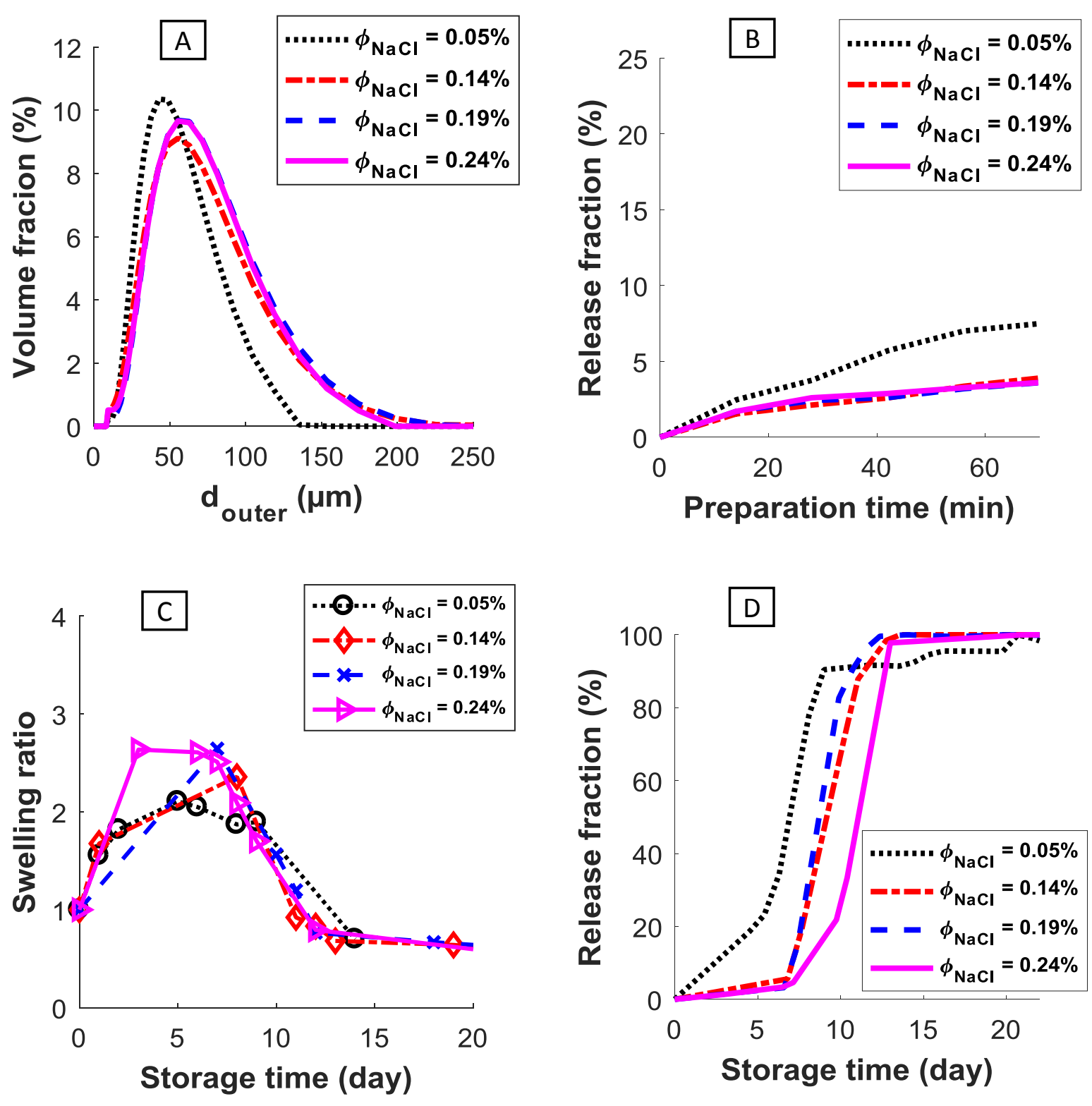

Fig. 11. Effect of salt fraction ( $\left.\phi_{\text {NaCl }}\right)$ on the: $A$ ) droplet size distribution during preparation, $B$ ) release during preparation, C) swelling ratio during storage, and D) release during storage. 

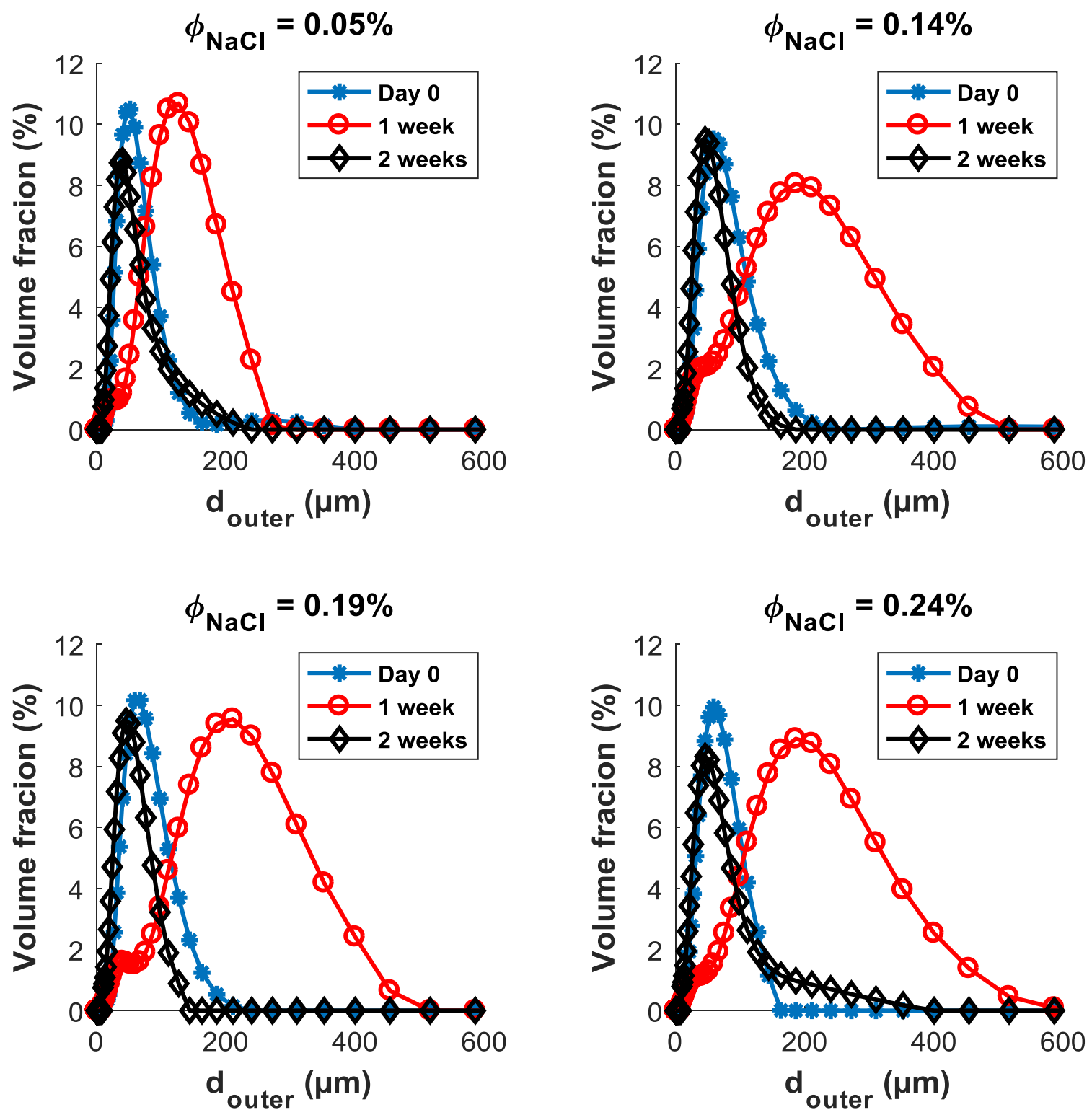

Fig. 12. Effect of the salt fraction $\left(\phi_{\text {NaCl }}\right)$ on the outer droplet size distribution during storage. 

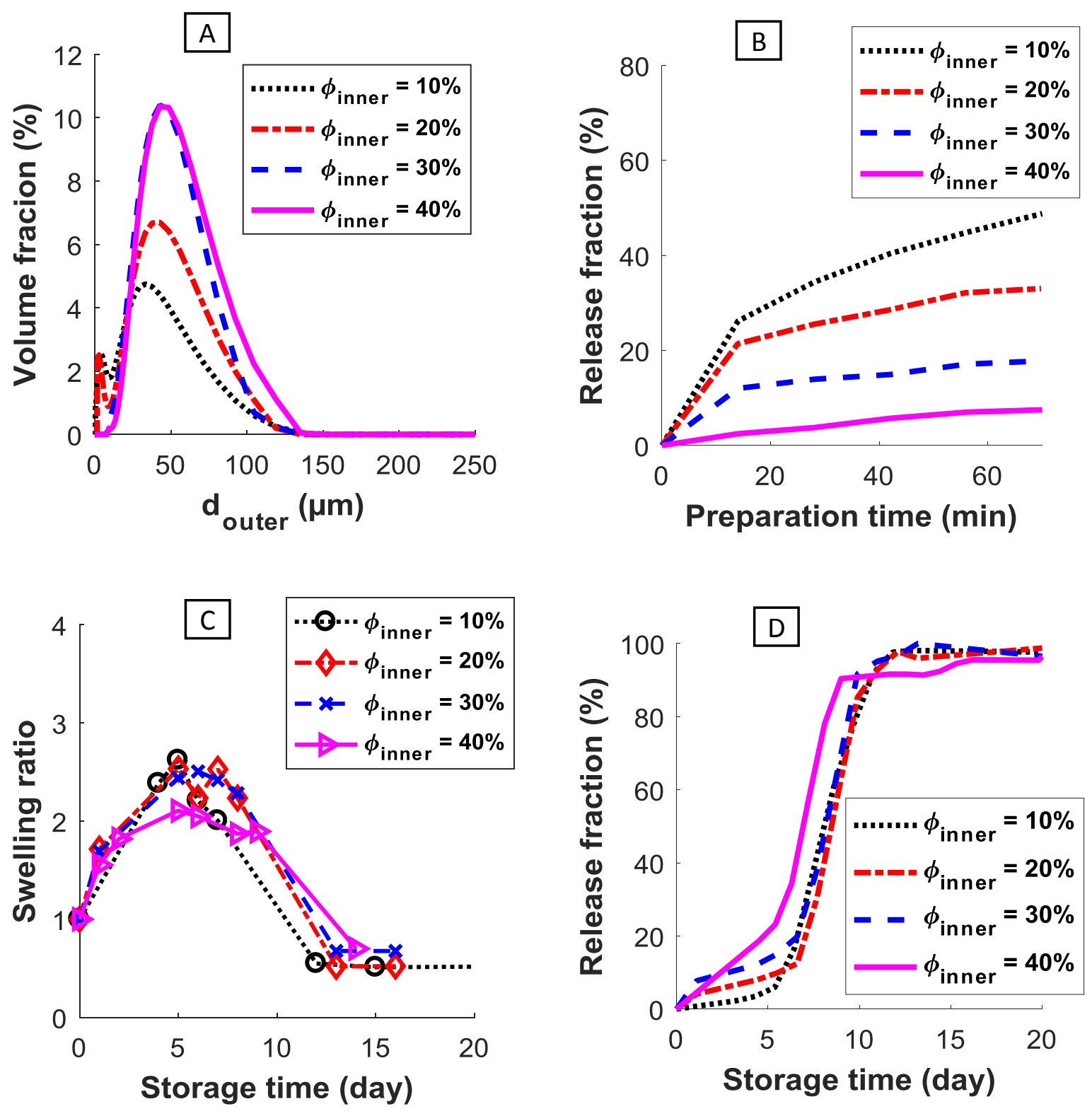

Fig. 13. Effect of the internal phase fraction ( $\left.\phi_{\text {inner }}\right)$ on: $\left.A\right)$ the outer droplet size distribution during preparation, $B$ ) the released fraction during preparation, C) the swelling ratio during storage, and D) the release of salt during storage. 

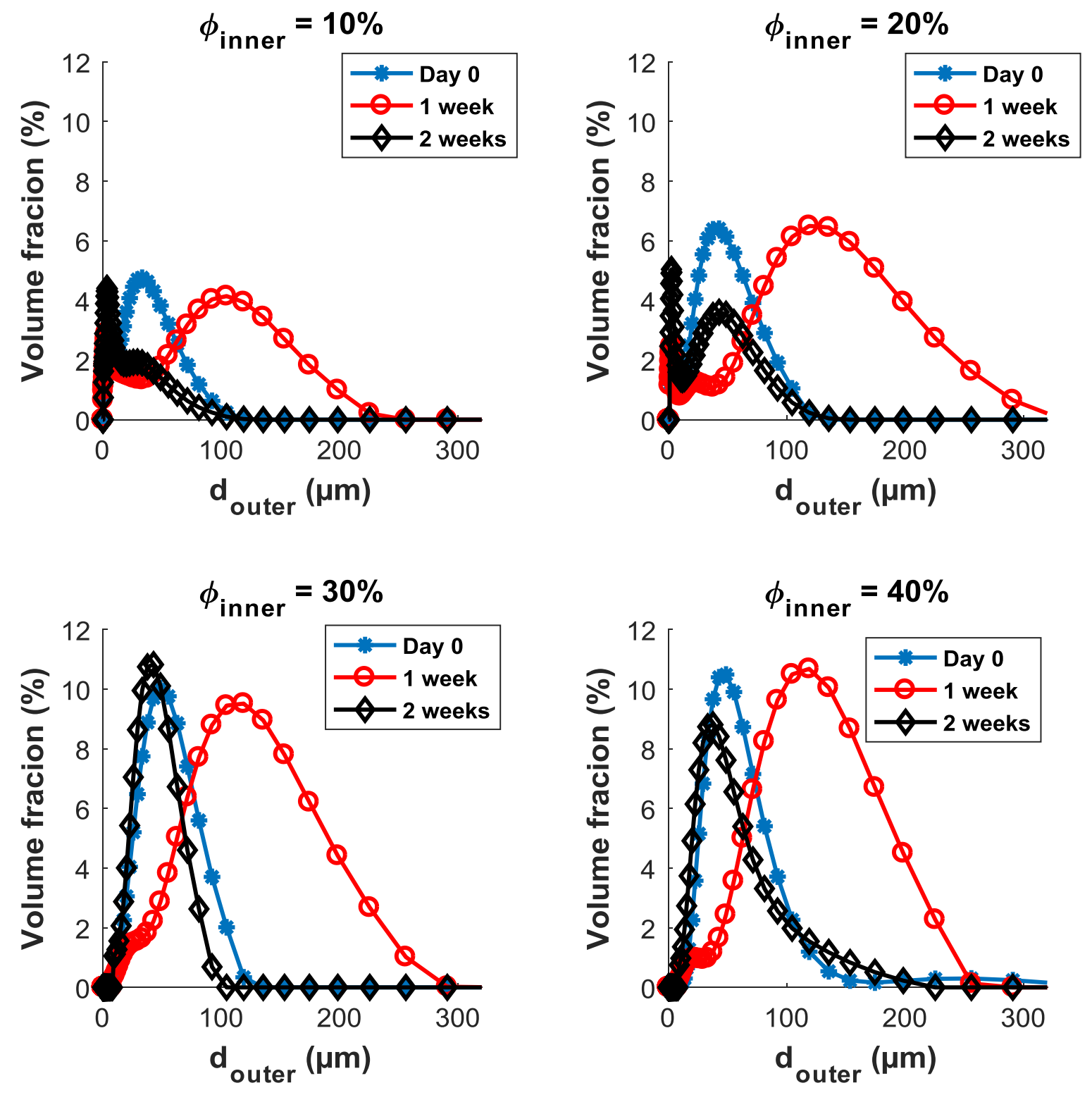

Fig. 14. Effect of internal phase fraction ( $\phi_{\text {inner }}$ ) on the droplet size distribution during storage. 

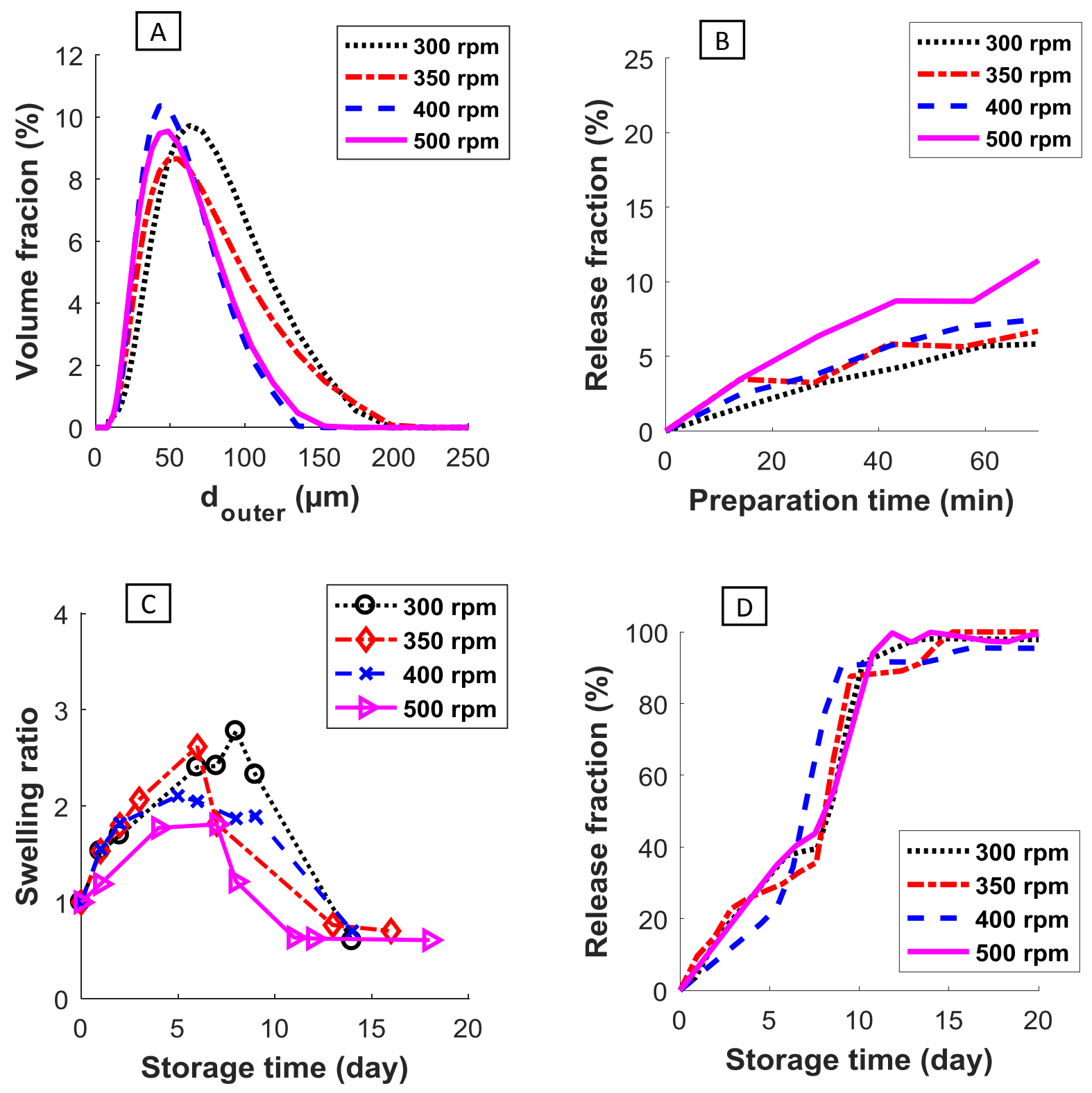

Fig. 15. Effect of the stirring rate $\left(\omega_{R}\right)$ on: $\left.A\right)$ the outer droplet size distribution during preparation, $B$ ) the released fraction during preparation, $C)$ the swelling ratio during storage, and $D)$ the release of salt during storage. 

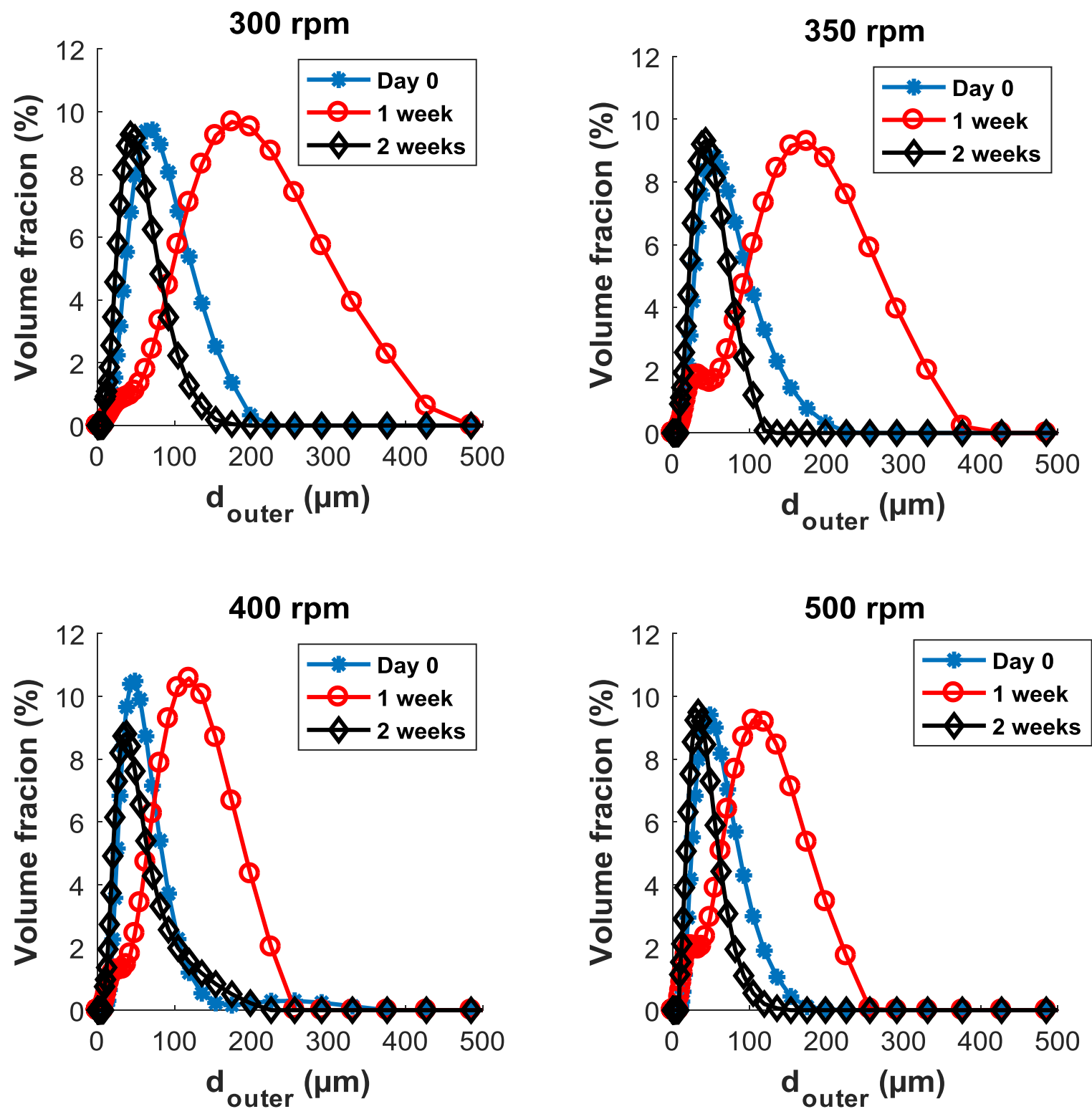

Fig. 16. Effect of the stirring rate $\left(\omega_{R}\right)$ on the outer droplet size distribution during storage. 